\title{
IL-12 enhances the natural killer cell cytokine response to Ab-coated tumor cells
}

\author{
Robin Parihar, ${ }^{1,2}$ Julie Dierksheide, ${ }^{3}$ Yan $\mathrm{Hu}^{2},{ }^{2}$ and William E. Carson ${ }^{1,2,3}$ \\ ${ }^{1}$ Department of Molecular Virology, Immunology, and Medical Genetics, \\ ${ }^{2}$ The Arthur G. James Comprehensive Cancer Center, and \\ ${ }^{3}$ Department of Surgery, The Ohio State University College of Medicine, Columbus, Ohio, USA
}

\begin{abstract}
The anti-tumor activity of recombinant mAb's directed against tumor cell growth receptors has generally been considered to result from direct antiproliferative effects, the induction of apoptosis, or possibly Ab-dependent cellular cytotoxicity mediated against tumor targets. However, it remains unclear to what degree these mechanisms actually aid in the clearance of Ab-coated tumor cells in vivo. We show here that $\mathrm{NK}$ cells secrete a distinct profile of potent immunostimulatory cytokines in response to dual stimulation with Ab-coated tumor cells and IL-12. This response could not be duplicated by costimulation with other ILs and was significantly enhanced in the presence of monocytes. Cytokine production was dependent upon synergistic signals mediated by the activating receptor for the Fc portion of IgG (FcrRIII) and the IL-12 receptor expressed on NK cells. Coadministration of Ab-coated tumor cells and IL-12 to BALB/c mice resulted in enhanced circulating levels of NK cell-derived cytokines with the capacity to augment anti-tumor immunity. These findings suggest that, in addition to mediating cellular cytotoxicity and apoptosis, the anti-tumor activity of mAb's might also result from activation of a potent cytokine secretion program within immune effectors capable of recognizing mAb-coated targets.
\end{abstract}

J. Clin. Invest. 110:983-992 (2002). doi:10.1172/JCI200215950.

\section{Introduction}

HER2/neu is a proto-oncogene that encodes a cell-surface transmembrane receptor with intracellular tyrosine kinase activity (1). Upon ligand binding, the HER2 receptor dimerizes with other members of the EGF-R family and initiates intracellular signals that result in proliferation. The HER2/neu gene is amplified in approximately $25 \%$ of human adenocarcinomas (2). Overexpression of this receptor leads to ligand-independent activation of the HER2 receptor kinase and aberrant epithelial cell proliferation. Breast cancer patients with this alteration frequently exhibit a worse histological grade, decreased relapse-free and overall survival, and altered sensitivity to standard chemotherapeutic regimens $(1,3)$. A murine $\mathrm{mAb}$ directed against the extracellular domain of the HER2 receptor (mu4D5) was developed in the hope that tumors exhibiting HER2-dependent growth might respond with reduced proliferation (4). Indeed, this $\mathrm{Ab}$ does inhibit the growth of HER2-positive tumor cells in

Received for publication May 17, 2002, and accepted in revised form Angust 30, 2002

Address correspondence to: William E. Carson, N924 Doan Hall, 410 West 10th Avenue, The Ohio State University College of Medicine, Columbus, Ohio 43210, USA. Phone: (614) 293-6306; Fax: (614) 688-4366; E-mail: carson-1@medctr.osu.edu. Conflict of interest: No conflict of interest has been declared. Nonstandard abbreviations used: Ab-dependent cellular cytotoxicity (ADCC); macrophage inflammatory protein- $1 \alpha$ (MIP-1 $\alpha$ ); antigen presenting cell (APC); IL-12 receptor (IL-12R); recombinant human (rhu); recombinant murine $(\mathrm{rmu})$; phycoerythrin (PE); protein tyrosine kinase (PTK). vitro and mediates regression of established tumors in a variety of animal models (5). Therapy with a recombinant, humanized form of this $\mathrm{Ab}$ (trastuzumab, trade name Herceptin) has shown efficacy in clinical trials, both as a single agent and when administered with chemotherapeutic drugs $(6,7)$. However, only $25-30 \%$ of patients with HER2-overexpressing malignancies will respond to these treatments. The ability to effectively combine Herceptin with other anti-tumor agents might be enhanced with a more complete understanding of its mechanism of action.

The binding of Herceptin to the HER2 receptor may lead to alterations in intracellular signaling and cell cycle kinetics within the target cell (8). In addition, Herceptin may also stimulate important immune responses such as $\mathrm{Ab}$-dependent cellular cytotoxicity (ADCC) and complement activation that may lead to direct tumor lysis (9). Indeed, it has been shown in a murine tumor xenograft model that the anti-tumor effects of Herceptin are dependent upon the presence of host immune cells that express receptors for the $\mathrm{Fc}$ portion of $\operatorname{IgG}(\mathrm{FcR})(10)$. Important ADCC-mediating effector cells that express $\mathrm{FCR}$ include monocytes/macrophages, resting and activated granulocytes, and NK cells. Although most immune cells coexpress both activating and inhibitory FcR's, NK cells are unique in that they constitutively express only the activating, low-affinity receptor FcyRIII (11). They also contain abundant cytolytic granules, prominently express cellular adhesion molecules, and constitutively display multiple cytokine receptors. Indeed, NK cell cytotoxicity against HER2-expressing tumor cells is 
markedly enhanced following treatment with IL-2 or IL-12 $(12,13)$. NK cells are also sources of potent immunostimulatory cytokines such as TNF- $\alpha$, GM-CSF, macrophage inflammatory protein- $1 \alpha$ (MIP$1 \alpha$ ), and IFN- $\gamma(11)$. Thus, while the anti-tumor activity of Herceptin has largely been attributed to direct antiproliferative and proapoptotic effects, we theorized that the clearance of Ab-coated tumor cells might be enhanced by the coadministration of immunologic adjuvants with the capacity to stimulate NK cell cytotoxicity and cytokine production.

IL-12 is an antigen presenting cell-derived (APCderived) cytokine that stimulates T cells and NK cells to secrete IFN- $\gamma$ and augments the proliferation and cytolytic activity of these cells (14). In addition to its critical role in the regulation of early inflammatory responses and promotion of the Th1-type repertoire, preclinical studies have suggested that IL-12 is an effective anti-cancer agent against various experimental malignancies. Work in numerous murine models has demonstrated that the anti-tumor effects of IL-12 are primarily mediated via the induction of IFN- $\gamma$ secretion by $\mathrm{T}$ and NK cells $(15,16)$. Of note, IL-12-induced IFN- $\gamma$ production has been shown to prevent mammary carcinogenesis in HER2/neu transgenic mice when IL-12 was administered alone or in combination with tumor cell- or dendritic cell-based vaccines $(17,18)$. Neutralization of IFN- $\gamma$ in this and other models strongly inhibits the anti-tumor activity of IL-12. Similarly, depletion of NK cells within hosts given exogenous IL-12 has been shown to attenuate the anti-tumor effect, suggesting a vital role for NK cells in the anti-tumor activity of IL-12 (19).

In the current report, we have examined the ability of IL-12 to enhance NK cell-mediated cytotoxicity and promote immunostimulatory cytokine secretion. We demonstrate that costimulation of pure NK cells or whole PBMCs with Herceptin-coated human breast cancer cells and IL-12 results in a unique cytokine secretion profile highlighted by abundant production of IFN- $\gamma$. Furthermore, we provide evidence that cytokine production in response to Herceptin-coated tumor cells and IL-12 is mediated by synergistic signals provided by Fc $\gamma$ RIII and the IL-12 receptor (IL-12R), and that this synergistic effect could not be duplicated by IL-2, IL-10, IL-15, or IL-18. These findings provide a strong rationale for the concurrent administration of Herceptin and IL-12 for the treatment of HER2-overexpressing malignancies and lend insight into the potential role of NK cells in the elimination of $\mathrm{Ab}$-coated tumor targets.

\section{Methods}

Cytokines and $A b$ 's. Recombinant human IL-12 (rhuIL12) and murine IL-12 (rmuIL-12) were kindly provided by Genetics Institute Inc. (Cambridge, Massachusetts, USA). rhuIL-2 with a specific activity of $1.53 \times 10^{7}$ $\mathrm{U} / \mathrm{mg}$ was obtained from Hoffman-LaRoche Inc. (Nutley, New Jersey, USA). rhuIL-15, rhuIL-18, and rhuIL-10 were purchased from PeproTech Inc. (Rocky Hill, New Jersey, USA). Cytokines were resuspended in
$1 \times$ PBS plus $0.1 \%$ BSA. The humanized anti-HER2 $\mathrm{mAb}$ Herceptin was kindly provided by Genentech Inc. (San Francisco, California, USA) (20). Purified F $\left(\mathrm{ab}^{\prime}\right)_{2}$ fragments of Herceptin were generated by pepsin digestion as previously described (21). Polyclonal huIgG was purchased from Sigma-Aldrich (St. Louis, Missouri, USA). For preliminary costimulation experiments, 96well flat-bottom plates were coated with huIgG by incubation with $100 \mu \mathrm{g} / \mathrm{ml}$ huIgG in $100 \mu \mathrm{l}$ of PBS overnight at $4^{\circ} \mathrm{C}$. Wells were then washed twice with $200 \mu \mathrm{l}$ fresh PBS and once with warm 10\% HAB medium consisting of heat-inactivated pooled human $A B$ serum (C-six Diagnostics Inc., Germantown, Wisconsin, USA), $100 \mathrm{U} / \mathrm{ml}$ penicillin, $100 \mu \mathrm{g} / \mathrm{ml}$ streptomyocin, and $0.25 \mu \mathrm{g} / \mathrm{ml}$ amphotericin, prior to use.

Cell lines. The SKBR3 (HER2-overexpressing) and MDA-468 (HER2-negative) human breast adenocarcinoma lines were obtained from American Type Culture Collection (Manassas, Virginia, USA). The MCF-

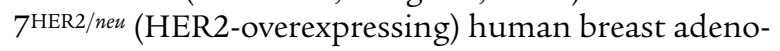
carcinoma was obtained from Jose Baselga (Hospital Universitari Vall D'Hebron, Barcelona, Spain). The MCF-7HER2/new cell line was created by transfection of parental MCF-7 cells with an expression cassette for human HER2/neu and culture in standard medium containing neomycin (22). A murine colon carcinoma line overexpressing human HER2/neu, CT-26 ${ }^{\mathrm{HER} 2 / n e u}$, and the parental line, CT-26, were kind gifts from P.T.P. Kaumaya (Ohio State University, Columbus, Ohio, USA) and were used for in vivo costimulation studies (23). Cancer cell lines were propagated in RPMI-1640 medium supplemented with $10 \%$ heat-inactivated FBS, $300 \mu \mathrm{g} / \mathrm{ml}$ L-glutamine, $100 \mathrm{U} / \mathrm{ml}$ penicillin, $100 \mu \mathrm{g} / \mathrm{ml}$ streptomycin, $0.25 \mu \mathrm{g} / \mathrm{ml}$ amphotericin $\mathrm{B}$, and 0.06 $\mathrm{mg} / \mathrm{ml}$ anti-PPLO agent (all from Life Technologies Inc., Rockville, Maryland, USA).

Mice. Female BALB/c mice between the ages of 5 and 7 weeks were purchased from The Jackson Laboratory (Bar Harbor, Maine, USA). STAT4 ${ }^{-/}$breeder mice on a B6 $\times 129$ strain background were a kind gift from James Ihle (St. Jude Children's Research Hospital, Memphis, Tennessee, USA), and females were used in experiments at 5-7 weeks of age (24). Age-matched wild-type $\mathrm{B} 6 \times 129$ mice (The Jackson Laboratory) were used as controls. Mice were maintained in the animal facility at the Ohio State University Comprehensive Cancer Center (CCC) with free access to food and water. All protocols were approved by the Ohio State University CCC Animal Care and Use Committee, and mice were treated in accordance with the institutional guidelines for animal care.

Isolation of human NK cells. PBMCs were isolated from fresh leukopacks (American Red Cross, Columbus, Ohio, USA) using Ficoll-Hypaque (Sigma-Aldrich) density gradient centrifugation. PBMCs were washed in RPMI-1640 (Life Technologies Inc.) and adhered overnight to plastic plates to eliminate the monocyte population. Nonadherent peripheral blood lymphocytes were further depleted of T cells, B cells, and mono- 
cytes using an $\mathrm{Ab}$ cocktail against epitopes of $\mathrm{CD} 3$, CD4, and HLA-DR, respectively, in conjunction with goat anti-mouse immunomagnetic beads, as previously described (25). In some experiments, NK cells were isolated directly from peripheral blood leukopacks by 30-minute incubation with RossetteSep cocktail (Stem Cell Technologies, Vancouver, British Columbia, Canada), followed by Ficoll-Hypaque density gradient centrifugation. Depleted peripheral blood lymphocytes were further stained with CD56-phycoerythrin (CD56PE) (Beckman Coulter, Fullerton, California, USA), washed, and sorted for a population containing both CD56 $6^{\text {dim }}$ and CD56 $6^{\text {bright }}$ (herein referred to as CD56 $6^{+}$ NK cells on a FACStar Plus cell sorter (Becton, Dickinson and Co., Franklin Lakes, New Jersey, USA). Sorted CD56 ${ }^{+}$NK cells were greater than $96 \%$ pure by FACStar analysis. For our in vitro assay, human PBMCs and NK cells were cultured in $10 \% \mathrm{HAB}$ medium.

In vitro coculture assay. Wells of a 96-well flat-bottom culture plate were coated with either the HER2-overexpressing cell line SKBR3, the HER2-negative cell line MDA-468, or a breast cancer cell line engineered to overexpress HER2/neu, MCF-7HER2/neu. Tumor cells $\left(5 \times 10^{4}\right)$ were grown to confluence overnight at $37^{\circ} \mathrm{C}$. The culture supernatant was aspirated the following day, and wells were treated with $100 \mu \mathrm{g} / \mathrm{ml}$ Herceptin for 1 hour at $37^{\circ} \mathrm{C}$ and then washed twice with warm medium to remove any unbound $\mathrm{Ab}$. Washing with warm medium had no appreciable effect on cell viability. Unfractionated PBMCs or purified CD $56^{+} \mathrm{NK}$ cells isolated from normal donors were added at $2 \times 10^{5}$ cells/well in $200 \mu \mathrm{l}$ medium in the presence of $10 \mathrm{ng} / \mathrm{ml}$ rhulL-12. Control wells contained tumor cells plus PBMCs or NK cells supplemented with medium alone, Herceptin alone, Herceptin $\mathrm{F}\left(\mathrm{ab}^{\prime}\right)_{2}$ fragment, or rhuIL-12 alone. Culture supernatants were harvested at the indicated timepoints and analyzed for levels of various cytokines (IFN- $\gamma$, TNF- $\alpha$, GM-CSF, and MIP- $1 \alpha$ ) by ELISA using commercially available $\mathrm{mAb}$ pairs (Endogen Inc., Woburn, Massachusetts, USA). Based on the manufacturer's guidelines, a standard sandwich ELISA for each human cytokine was developed, and cytokine concentrations were determined by the use of standard curve regression analysis as previously described (15). The detection limit for all ELISAs was $10-30 \mathrm{pg} / \mathrm{ml}$. All results are shown as the mean of triplicate wells \pm SEM.

Real-time PCR. Cells were harvested from culture and lysed with $500 \mu$ RNA lysis buffer. Total cellular RNA was isolated using RNeasy Mini Kits (Qiagen Inc., Valencia, California, USA), and cDNA was generated with random hexamer primers and MMLV-RT according to manufacturer recommendations (Life Technologies Inc.). Using the generated cDNA as template, real-time PCR for human IFN- $\gamma$ transcript was performed as multiplex reactions with primer and probe sets specific for the cytokine transcript and an 18S rRNA internal control (PE Applied Biosystems, Foster City, California, USA). Reactions were performed using an ABI Prism 7700 sequence detector, and data were analyzed with Sequence Detector (version 1.6) software (PE Applied Biosystems). Data were analyzed according to the comparative $\mathrm{C}_{\mathrm{T}}$ method using $18 \mathrm{~S}$ internal control transcript levels to normalize differences in sample loading and preparation. Results are semiquantitative and represent the fold difference in transcript levels in a particular sample as compared with levels from unstimulated cells.

Administration of Herceptin plus IL-12 to mice. CT-26 and CT-26 ${ }^{\mathrm{HER} 2 / \text { nen }}$ cells were harvested from culture via mild trypsinization, washed once with sterile PBS, and incubated at $4^{\circ} \mathrm{C}$ in PBS plus $10 \%$ FBS at $1 \times 10^{7}$ cells $/ \mathrm{ml}$ with either Herceptin $(1 \mathrm{mg} / \mathrm{ml})$ or normal huIgG (1 $\mathrm{mg} / \mathrm{ml}$ ) for 45 minutes. Cells were then washed twice in sterile PBS, and $4 \times 10^{6}$ cells in PBS were given intraperitoneally to each mouse concurrently with a separate intraperitoneal injection of $1 \mu \mathrm{g}$ muIL-12 in PBS. Each group consisted of ten wild-type BALB/c mice, and control groups received injections of IgG-treated tumor alone, IgG-treated tumor plus IL-12, or Herceptin-coated tumor alone. Serum was harvested from each mouse at 6,12, and 24 hours and analyzed for cytokine levels by ELISA. Mice were immediately euthanized, and splenocytes were harvested for use in our in vitro coculture assay using CT-26 and CT-26 ${ }^{\mathrm{HER} 2 / \text { neu }^{2}}$ cells as the HER2 ${ }^{-}$and HER $2^{+}$tumor cell lines, respectively. Culture supernatants were harvested after 24 hours and analyzed for levels of muIFN- $\gamma$ by ELISA (R\&D Systems Inc., Minneapolis, Minnesota, USA).

Statistics. Statistical analysis of ELISA cytokine levels was performed using the paired Student $t$ test, with $P<0.05$ considered significant.

\section{Results}

NK cells costimulated with immobilized IgG and IL-12 produce large quantities of IFN- $\gamma$. Our laboratory has previously demonstrated that NK cells can mediate ADCC against Ab-coated tumor targets and that the lytic activity of the NK cell compartment is markedly enhanced by activation with IL-2 or IL-15 (12). Our recent observations show that ADCC mediated by breast cancer patient PBMCs (26) against HER2-overexpressing cell lines is also markedly enhanced following culture of effectors with IL-12, and the observed lytic activity is comparable to that obtained with IL-2 (data not shown). We were next interested in determining the ability of IL-12 to enhance NK cell cytokine production in the presence of immobilized huIgG, as the NK cell response to this stimulus has not been previously characterized. Purified NK cells cultured for 72 hours in the presence of both immobilized huIgG and rhuIL-12 produced large amounts of IFN- $\gamma$, while NK cells stimulated with immobilized IgG alone or IL-12 alone produced negligible amounts of this cytokine (Figure 1a). Further experiments revealed a clear IL-12 dose-dependent effect on secretion of IFN- $\gamma$. Detectable NK cytokine secretion could be achieved upon costimulation with only $0.1 \mathrm{ng} / \mathrm{ml} \mathrm{IL-12}$. However, optimal production of IFN- $\gamma$ required higher 
a

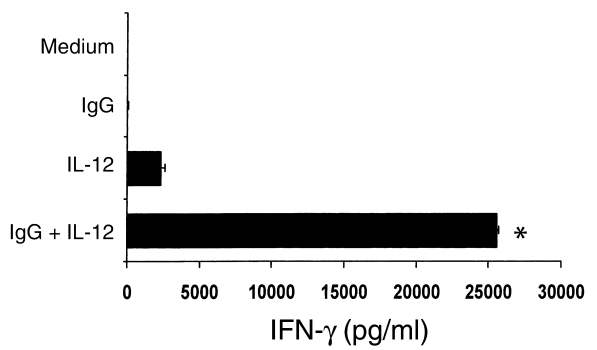

b

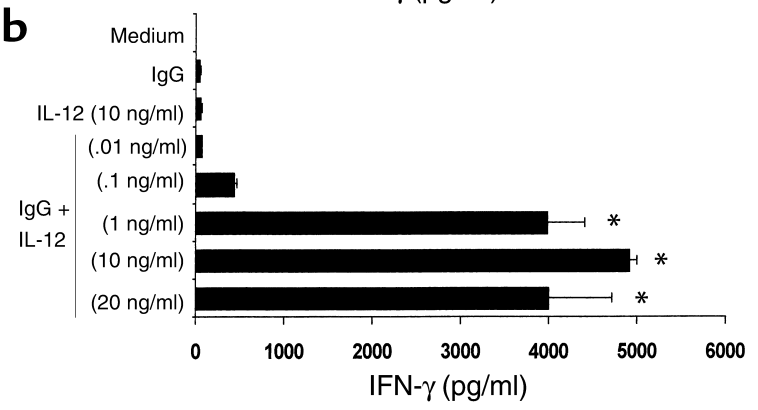

C

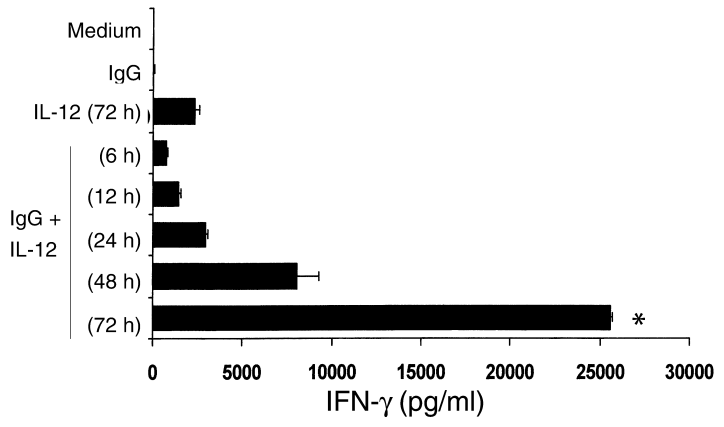

Figure 1

NK cells costimulated with immobilized IgG and IL-12 secrete high levels of IFN- $\gamma$. (a) Human NK cells were cultured on wells precoated with hulgG. rhulL-12 was added at a concentration of $10 \mathrm{ng} / \mathrm{ml}$. Control wells consisted of NK cells cultured with medium alone (Medium), immobilized hulgG alone (IgG), or rhulL-12 alone (IL-12). Culture supernatants were harvested after 72 hours and analyzed for IFN- $\gamma$ content by ELISA. (b) Human NK cells were cultured on immobilized hulgG in varying concentrations of rhulL-12 $(0.01-20 \mathrm{ng} / \mathrm{ml})$, and supernatants were harvested after 72 hours. (c) In time-course experiments, NK cells were cultured with $10 \mathrm{ng} / \mathrm{ml} \mathrm{IL-12}$ on immobilized hulgG. Supernatants were harvested at varying times (6-72 hours). ${ }^{*} P<0.001$ versus medium, IgG, and IL-12.

levels of IL-12 (Figure 1b). While levels of IFN- $\gamma$ were detected after 6 hours in culture, peak accumulation did not occur until 72 hours after stimulation (Figure 1c). In order to confirm the specific effects of IL-12, other cytokines were examined for their ability to act as costimuli for NK cell cytokine production. Interestingly, markedly lower levels of IFN- $\gamma$ were obtained when NK cells were stimulated with immobilized IgG in combination with IL-2, IL-10, IL-15, IL-18 (Figure 2 ), or IFN- $\alpha$ (not shown). Thus, under the conditions of this assay, IL-12 appears to be the most effective costimulus for NK cell secretion of IFN- $\gamma$.

Herceptin and IL-12 provide a potent costimulus for NK cell cytokine production. Culture of human NK cells in the presence of IL-12 and immobilized anti-tumor
mAb's such as Herceptin or rituximab (a humanized $\mathrm{mAb}$ recognizing the $\mathrm{CD} 20 \mathrm{~B}$ cell antigen) (27) also resulted in significant production of IFN- $\gamma$ over the course of 72 hours (data not shown). In order to extend these observations in the context of combination therapy using Herceptin plus cytokine, we proceeded to develop an in vitro assay that might better reflect the tumor microenvironment.

HER2-overexpressing (SKBR3 and MCF-7 ${ }^{\mathrm{HER} 2} / \mathrm{neu}$ ) and HER2-negative (MDA-468) cell lines were grown to confluence in flat-bottom wells and coated with Herceptin. Each well was then supplemented with IL-12 and purified human NK cells. Herceptin-coated tumor cells served as a potent stimulus for NK cell cytokine production, provided that IL-12 was present in the culture medium (Figure $3 \mathrm{a}$ ). In contrast, there was minimal production of IFN- $\gamma$ in wells containing HER2-negative cells, regardless of the presence of IL-12. Similar results were obtained for a second pair of HER2-positive and -negative cell lines (SKBR3 and HT-1080, respectively). A time-course study revealed that NK cell production of IFN- $\gamma$ began within 12 hours of exposure to Herceptincoated HER2-positive cells (Figure 3b). Interestingly, a small amount of IFN- $\gamma$ was secreted in response to the HT-1080 cell line. Flow cytometric analysis revealed lowlevel surface overexpression of the HER2 antigen on this cell line (not shown), suggesting that combination therapy with Herceptin and IL-12 might be effective in patients whose tumors express modest levels of HER2. This represents a significant finding, as Herceptin treatments to date have shown efficacy only in patients whose tumors have high-level expression of the HER2 antigen (8). Analysis by real-time RT-PCR revealed a 35fold increase in cytokine transcript in NK cells costimulated with Herceptin-coated tumor cells and IL-12 (Figure 3c). In contrast, IFN- $\gamma$ transcript induction ver-

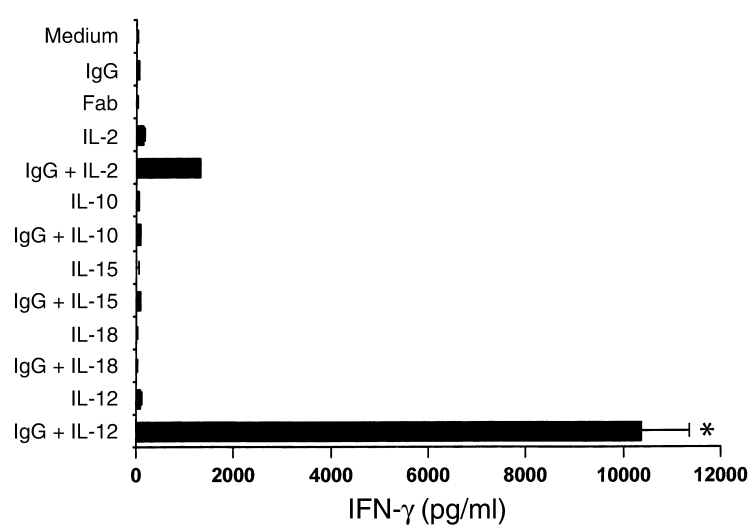

\section{Figure 2}

IL-12 is the most effective costimulus with immobilized IgG for IFN- $\gamma$ production by NK cells. Purified NK cells were cultured on immobilized hulgG in the presence of various human cytokines (IL-2, IL-10, $\mathrm{IL}-15, \mathrm{IL}-18$, and IL-12) at $10 \mathrm{ng} / \mathrm{ml}$. Standard controls were also included. As a further control, some wells were coated with human $\mathrm{F}\left(a b^{\prime}\right)_{2}$ fragment prior to the addition of NK cells (Fab). Culture supernatants were harvested after 72 hours and analyzed for IFN- $\gamma$ content by ELISA. ${ }^{*} P<0.005$ versus all conditions shown. 
sus an HER2-negative cell line was less than twofold for all conditions (not shown).

Synergistic cytokine secretion induced by IgG and IL-12 is mediated through distinct pathways. In order to elucidate the mechanism by which Herceptin and IL-12 act in synergy to induce cytokine secretion by NK cells, both IL-12 and IgG signaling pathways were examined. STAT4 is an important intracellular signaling molecule and transcription factor responsible for mediating IL-12-induced IFN- $\gamma$ secretion by $T$ cells and NK cells (24). Abrogation of STAT4-mediated signaling has been shown to dramatically affect the transcription of IL-12-stimulated genes. Splenocytes from wild-type mice and STAT4-deficient $\left(S T A T 4^{-/}\right)$mice were plated onto wells precoated with immobilized murine IgG and costimulated with murine IL-12. Whereas wild-type splenocytes displayed synergistic IFN- $\gamma$ production in response to immobilized IgG and IL-12, cells from STAT4 ${ }^{-/}$mice were unable to secrete IFN- $\gamma$ in response to stimulation with either IL-12 alone or with IL-12 plus immobilized IgG (Figure 4a). Importantly, cytokine secretion in response to immobilized IgG alone, while low, was not affected in STAT4 ${ }^{-/-}$cells.

To further clarify the contribution of IgG-induced signaling to cytokine secretion, specific inhibitors of intracellular signaling were employed in our in vitro costimulation assay. Freshly isolated human PBMCs were plated with rhuIL-12 on wells precoated with huIgG in the presence of piceatannol, a specific inhibitor of Syk and ZAP-70 kinases (28). Inhibition of IgG-mediated signaling by piceatannol dramatically reduced IFN- $\gamma$ secretion by costimulated PBMCs (Figure 4b). Taken together, these results suggest that cytokine secretion by NK cells in response to immobilized Herceptin and IL-12 is critically dependent upon these two distinct signals relayed from the cell surface to the nucleus.

PBMCs secrete greater amounts of cytokines than do purified $N K$ cells in response to Herceptin-coated tumor cells and IL-12. We next proceeded to determine whether there were quantitative differences in cytokine secretion between pure NK cells and whole PBMCs in response to costimulation with Herceptin-coated tumor cells and IL-12. The use of PBMCs in our coculture assay might provide a more accurate picture of the cytokine levels to be found within the tumor microenvironment following systemic administration of Herceptin and IL-12. Surprisingly, $2 \times 10^{5}$ PBMCs (consisting of approximately $12 \% \mathrm{NK}$ cells) consistently secreted greater amounts of IFN- $\gamma$ in response to Herceptin-coated HER2-overexpressing cells in the presence of IL- 12 than did $2 \times 10^{5}$ purified NK cells (Figure 5a). Large quantities of TNF- $\alpha$, GM-CSF, and MIP- $1 \alpha$ were also secreted by PBMCs in response to costimulation (Figure 5, b-d). These findings suggested that either a compartment other than NK cells was directly responsible for cytokine secretion in response to Herceptin and IL-12 costimulation, or the monocyte compartment might act to enhance the NK cell production of cytokines, or both. To help clarify these issues, cytokine production by specific immune effectors was examined.
Both NK and T cells secrete IFN- $\gamma$ in response to Herceptincoated tumor cells and IL-12. Having observed consistently higher levels of cytokine secretion by a whole PBMC population than by pure NK cells in our coculture model, we next examined which cellular compartment could be responsible for the additional cytokine secretion. We used flow cytometry to analyze intracellular
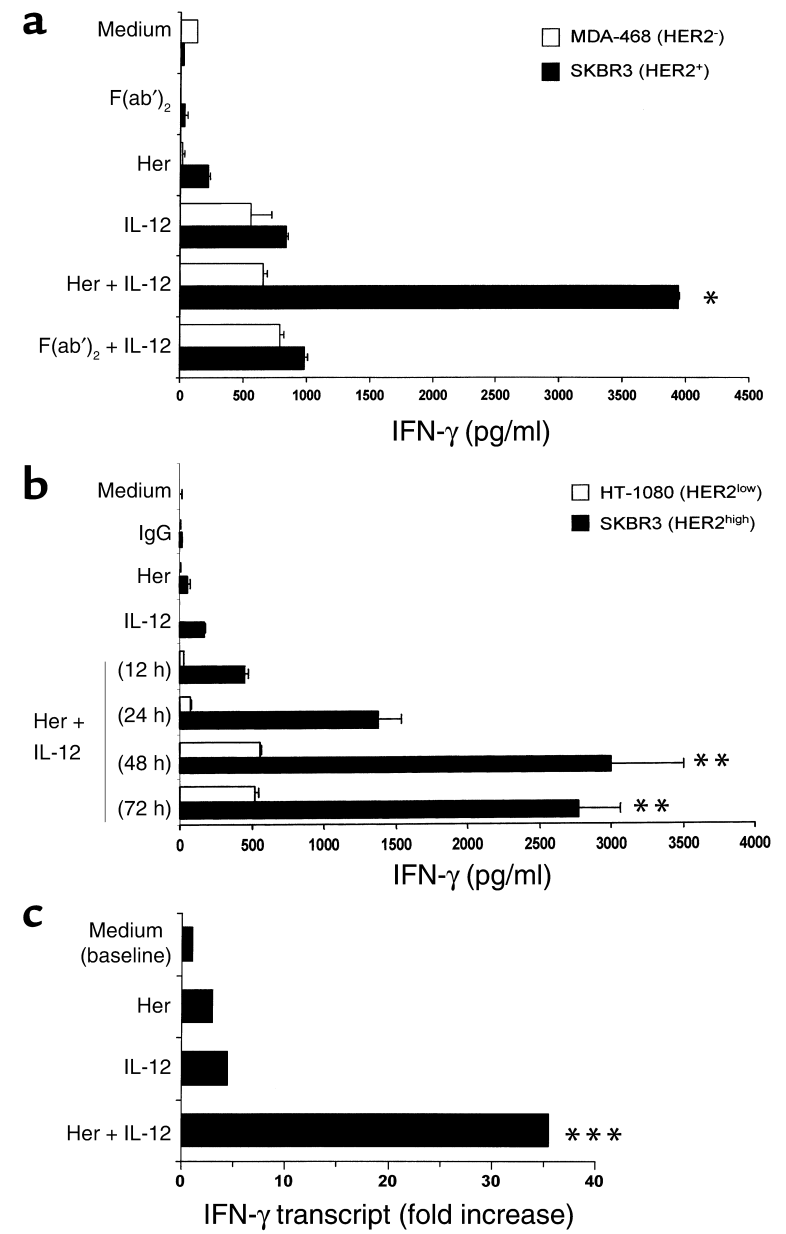

Figure 3

NK cells secrete large quantities of IFN- $\gamma$ in response to Herceptincoated human breast cancer cells and IL-12. (a) The MDA-468 and SKBR3 cell lines were cultured with purified NK cells in our in vitro coculture assay. Control wells contained tumor cells and NK cells supplemented with medium alone, Herceptin alone (Her), or rhulL12 alone. As a further control, some wells were treated with Herceptin $\mathrm{F}\left(\mathrm{ab} \mathrm{b}^{\prime}\right)_{2}$ fragment prior to the addition of NK cells. Culture supernatants were harvested after 72 hours and analyzed for IFN- $\gamma$ content by ELISA. ${ }^{*} P<0.01$ versus medium, $F\left(a b^{\prime}\right)_{2}$, Herceptin, IL-12, Her + IL-12 (MDA-468), and F( $\left.\mathrm{ab}^{\prime}\right)_{2}+\mathrm{IL}-12$ (MDA-468 and SKBR3). (b) The SKBR3 (HER2 high-level expression) and HT-1080 (HER2 low-level expression) human breast cancer cell lines were used in our in vitro coculture assay. Supernatants were harvested at the indicated times (12-72 hours). ${ }^{*} P<0.05$ versus medium, IgG, Herceptin, IL-12 (HT-1080 and SKBR3), and Her + IL-12 (HT-1080). (c) NK cells cultured in our in vitro coculture assay were harvested after 72 hours and processed for real-time PCR analysis of the indicated transcript (see Methods). Results are given as fold increase in cytokine transcript over baseline versus the SKBR3 cell line. ${ }^{*}{ }^{*} P<0.05$ versus medium, Her, and IL-12. 

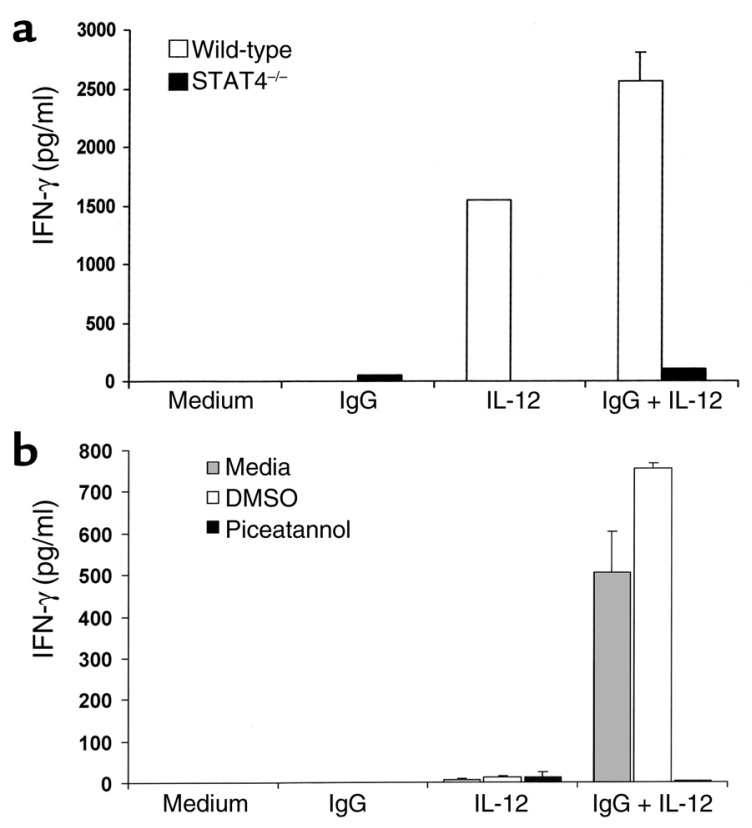

Figure 4

Both IL-12 receptor and Fc receptor signaling contribute to NK cell cytokine secretion following costimulation with IgG and IL-12. (a) Splenocytes from STAT4 ${ }^{-1-}$ mice or wild-type controls were cultured in the presence of $10 \mathrm{ng} / \mathrm{ml} \mathrm{rmulL-12}$ on plates precoated with 100 $\mu \mathrm{g} / \mathrm{ml}$ mulgG. Control wells contained splenocytes cultured with medium alone, mulgG alone, or rmulL-12 alone. Culture supernatants were harvested after 72 hours and analyzed for mulFN- $\gamma$. (b) Human PBMCs were cultured overnight in medium containing $30 \mu \mathrm{g} / \mathrm{ml}$ piceatannol. PBMCs were then cultured with $10 \mathrm{ng} / \mathrm{ml}$ rhulL-12 on plates precoated with hulgG. Standard controls were included. Additional controls included wells containing PBMCs pretreated with medium alone or with medium containing DMSO (the solvent for piceatannol) alone. Culture supernatants were harvested at 72 hours and analyzed for hulFN- $\gamma$.

levels of IFN- $\gamma$ protein induced within specific cell types in response to Herceptin-coated tumor cells and IL-12. As expected, NK cells secreted large amounts of IFN- $\gamma$ in response to Herceptin and IL-12 (Figure 6a). However, within the context of a PBMC population, T lymphocytes also secreted modest amounts of IFN- $\gamma$ (Figure $6 \mathrm{~b})$. No detectable IFN- $\gamma$ secretion by B cells, monocytes, or any cellular subset was observed in response to an HER2-negative cell line (not shown). The data in Figure $6 \mathrm{a}$ indicate that stimulation of NK cells with immobilized Herceptin or IL-12 might lead to significant levels of intracellular IFN- $\gamma$. However, permeabilization of NK cells by the addition of a mild detergent ( $1 \%$ SDS) to the immobilized IgG assays did not result in an appreciable increase in detection of IFN- $\gamma$ (not shown).

To further investigate the possibility that NK cell cytokine secretion was being affected by other cell types within the PBMC population, and based on the potential for interaction between NK cells and monocytes through cytokine signaling (29), we analyzed the effect of monocyte depletion in our coculture model. The removal of monocytes from the PBMC population severely attenuated IFN- $\gamma$ secretion by NK and T cells
(Figure 7a). This attenuation could be reversed upon return of monocytes to the coculture, even at as little as $25 \%$ of their original numbers (Figure 7b). Minimal IFN- $\gamma$ secretion was detected in cocultures that included the pure monocyte population only, regardless of culture conditions (not shown), further indicating that the role of the monocyte/macrophage compartment is to provide critical signals that promote NK cell cytokine secretion in response to Herceptin plus IL-12 costimulation.

IL-12 enhances in vivo cytokine production in response to Herceptin-coated tumor cells. In order to confirm cytokine secretion in vivo following costimulation with Herceptin-coated tumor cells and IL-12, we designed a costimulation assay in which Herceptin-coated, HER2expressing murine tumor cells and IL-12 were coadministered to naive mice. Administration of Herceptin-coated tumor cells and IL-12 resulted in increased serum levels of IFN- $\gamma$ and TNF- $\alpha$ as early as 12 hours after administration (Figure 8). Furthermore,
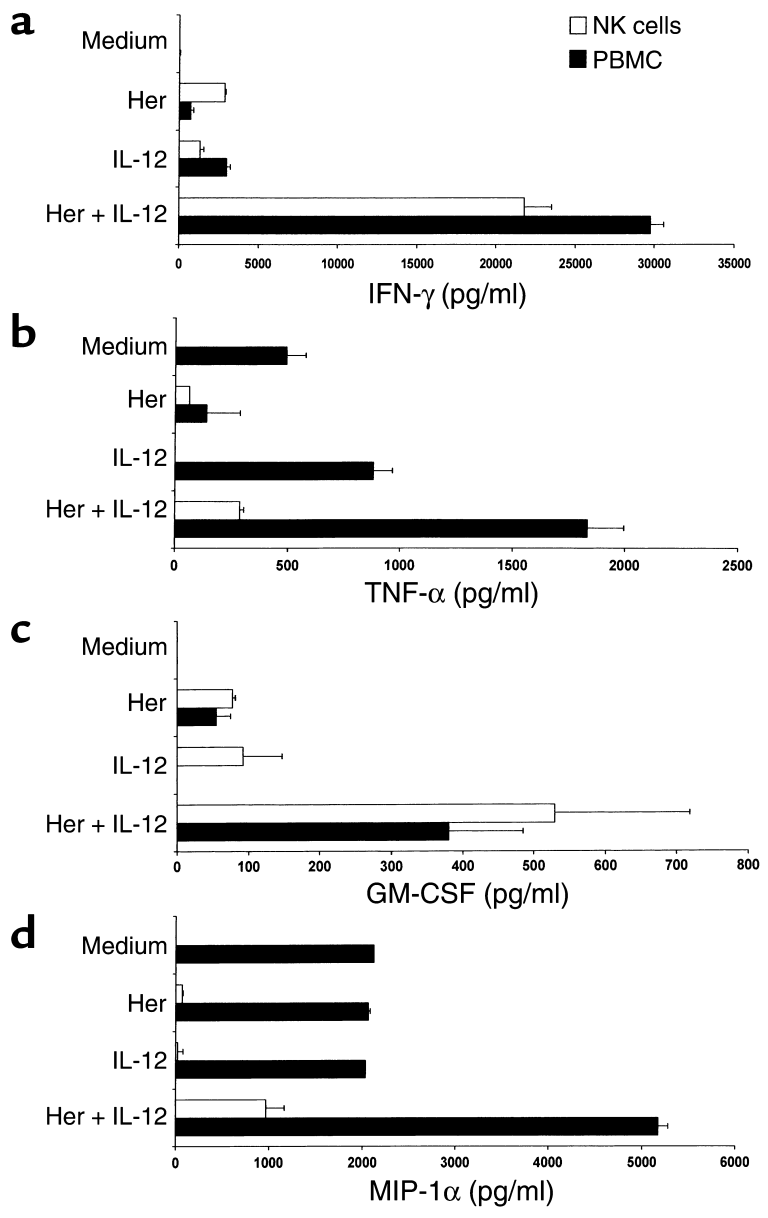

Figure 5

PBMCs secrete higher levels of immunostimulatory cytokines than do pure NK cells in response to Herceptin-coated human breast cancer cells and IL-12. The SKBR3 cell line was cultured with purified NK cells or a whole PBMC population in our in vitro coculture assay. Standard controls were also included. Culture supernatants were harvested after 72 hours and analyzed for (a) IFN- $\gamma(\mathbf{b})$ TNF- $\alpha$ (c) GM-CSF, and (d) MIP- $1 \alpha$. 


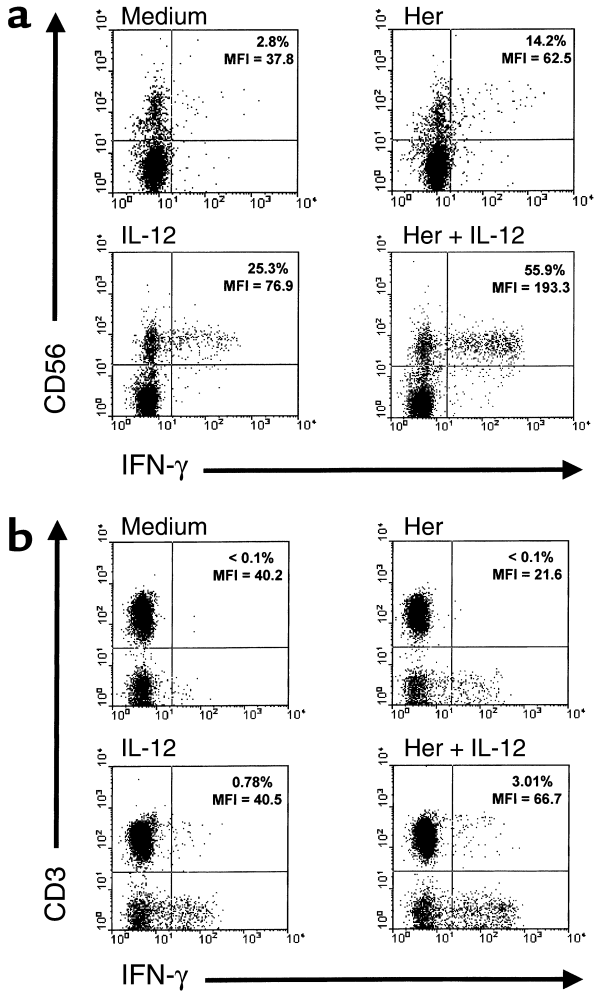

Figure 6

Both NK cells and T cells exhibit significant production of IFN- $\gamma$ in response to Herceptin and IL-12 costimulation. The MDA-468 and SKBR3 cell lines were cultured with PBMCs within our in vitro coculture assay. Standard controls were also included. Brefeldin A (10 $\mu \mathrm{g} / \mathrm{ml}$ ) was included in all culture wells in order to halt protein trafficking from the Golgi. After 6 hours of culture, cells were harvested and labeled for surface expression of either CD56, CD3, CD20, or CD14 with PE-labeled mAb's. Cells were then permeabilized, fixed, and stained for intracellular IFN- $\gamma$ protein with an FITC-labeled $\mathrm{mAb}$. Shown are representative dot plots of the PBMC population (vs. the SKBR3 cell line) stained for IFN- $\gamma$ production within (a) CD $56^{+} \mathrm{NK}$ cells and (b) $\mathrm{CD}^{+} \mathrm{T}$ cells. The percentage of cells secreting IFN- $\gamma$ along with the mean fluorescent intensity (MFI) is indicated within each plot. The fluorescent intensity of cells stained with isotype control Ab's routinely fell within the first log (not shown).

only splenocytes derived from mice stimulated with Herceptin-coated tumor cells plus IL-12 secreted IFN- $\gamma$ in response to in vitro restimulation with Herceptin and IL-12 in our coculture assay (data not shown). These results verify that an IL-12 costimulus can mediate significant cytokine production in vivo within the context of anti-HER2/neu mAb therapy.

\section{Discussion}

In the current report, we provide evidence that IL-12 and tumor-bound Herceptin synergize to induce potent cytokine secretion by NK cells. The combination of IL-12 with Herceptin stimulated NK cells to secrete abundant quantities of IFN- $\gamma$, MIP- $1 \alpha$, TNF- $\alpha$, and GM-CSF, a result that could not be duplicated by stimulation with IL-2, IL-10, IL-15, or IL-18. Experiments with signaling inhibitors showed that this unique cytokine secretion profile was critically dependent upon distinct, yet synergistic, signals mediated by IL-12R and FcR expressed on the surface of NK cells. Interestingly, whole PBMCs were more effective producers of IFN- $\gamma$ than were purified NK cells, an effect that was dependent upon the presence of monocytes. Furthermore, we have shown that administration of IL-12 with Herceptin in vivo resulted in enhanced circulating levels of cytokines in a murine tumor model. These results suggest that administration of IL-12 can uniquely enhance the immune response to Herceptincoated tumor cells.

Crosslinking of FcyRIII (CD16) on NK cells by immobilized IgG alone is a poor inducer of NK cell cytokine secretion and lytic activity. Previous studies have demonstrated that engagement of FcyRIII must be accompanied by specific costimulatory signals in order to obtain maximal enhancement of NK cell effector functions. Cassatella et al. reported that IL-2 can synergize with crosslinked CD16 to induce a rapid rise in intracellular $\mathrm{Ca}^{2+}$ and upregulate expression of IFN- $\gamma$ and TNF- $\alpha$ mRNA within NK cells (30). However, cytokine production at the protein level was not examined. More recently, IL-12 has been shown to augment
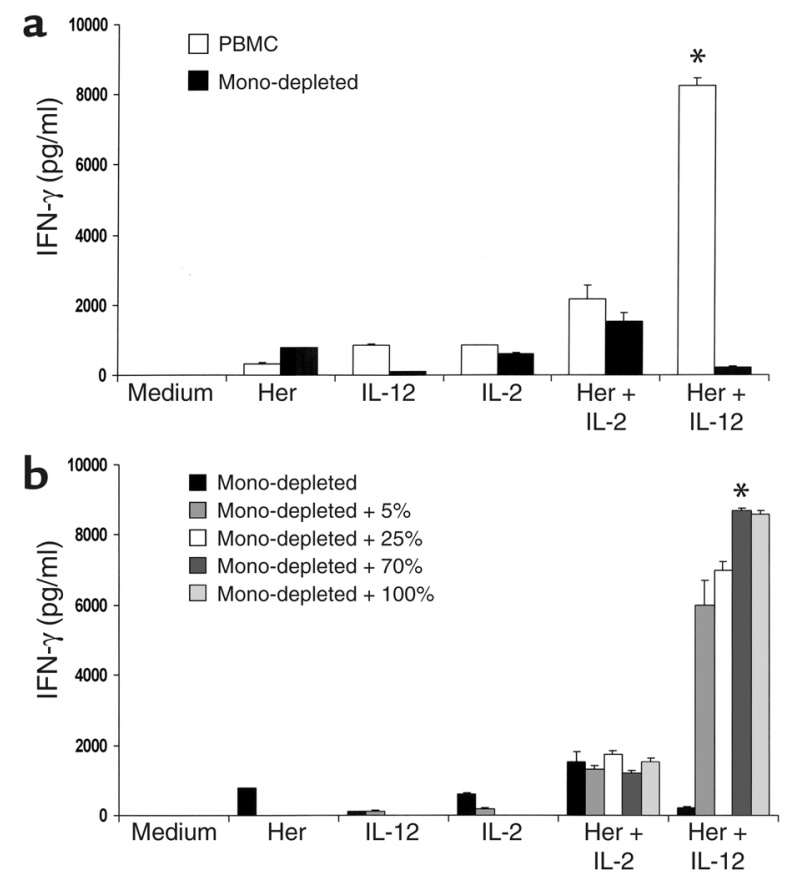

Figure 7

Monocyte depletion severely attenuates IFN- $\gamma$ secretion by PBMCs stimulated with Herceptin-coated tumor cells and IL-12. (a) A whole PBMC population (PBMC; $12 \% \mathrm{CD}_{14}{ }^{+}$cells), a monocyte-depleted PBMC population (Mono-depleted; $<0.1 \% \mathrm{CD} 14^{+}$cells), and a pure monocyte population (not shown; $95 \% \mathrm{CD}_{14}{ }^{+}$cells) were cultured with the SKBR3 cell line in our in vitro coculture assay with either $10 \mathrm{ng} / \mathrm{ml}$ rhulL-12 or rhulL-2. Standard controls were also included. (b) The monocyte-depleted population was "replenished" with various amounts (percent of original) of pure monocytes. Culture supernatants were harvested at 72 hours and analyzed for IFN- $\gamma$ content. ${ }^{*} P<0.05$ versus medium, Herceptin, IL-12, IL-2, Herceptin + IL-2, and Herceptin + IL-12 (monocyte-depleted). 

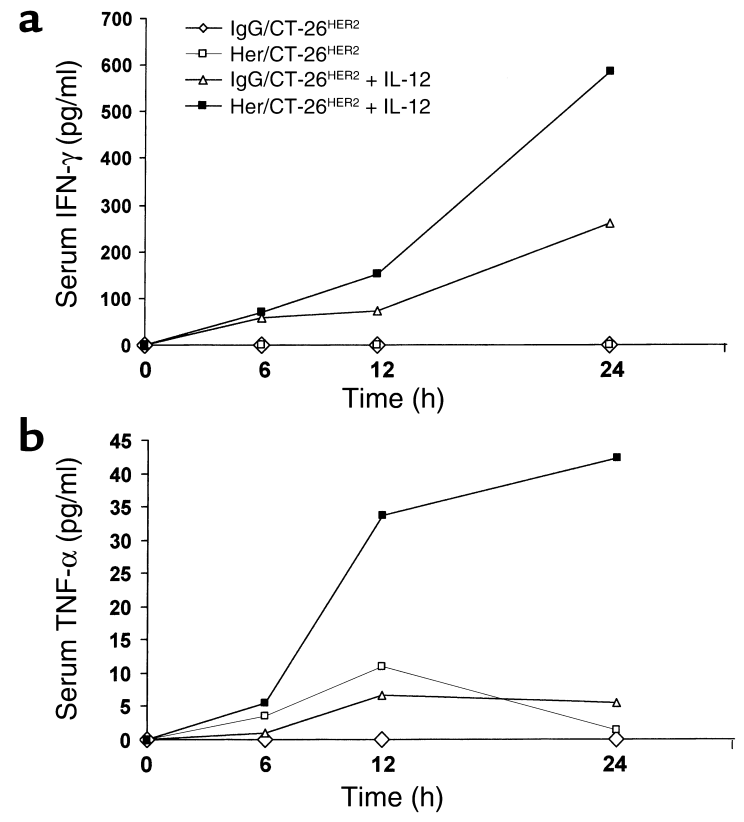

\begin{abstract}
Figure 8
Coadministration of Herceptin-coated tumor cells and IL-12 results in increased circulating levels of IFN- $\gamma$ in vivo. Herceptin-coated $\mathrm{CT}-26^{\mathrm{HER} 2 / \text { пеи }}$ murine tumor cells were injected intraperitoneally concurrently with $1 \mu \mathrm{g}$ mulL-12 ( $\left.\mathrm{Her} / \mathrm{CT}-26^{\mathrm{HER} 2}+\mathrm{IL}-12\right)$. Control groups included mice $(n=10)$ that received normal hulgG-treated tumor cells only (IgG/CT-26 $\left.{ }^{\text {HER } 2}\right)$, Herceptin-coated tumor cells only (Her/CT-26 HER2), or hulgG-treated tumor cells plus IL-12 (IgG/CT$26^{\text {HER2 }}+$ IL-12). Serum was harvested from each mouse at 6,12 , and 24 hours and analyzed by ELISA for levels of IFN- $\gamma$ and TNF- $\alpha$. Use of the parental CT-26 cell line in this assay did not result in cytokine production by any condition examined (not shown).
\end{abstract}

in vitro NK cell cytotoxicity against tumor cells treated with a CD16/CD30 bispecific mAb (31). Interestingly, this enhancement of mAb-mediated tumor lysis was achieved with concentrations of IL-12 that were considerably lower than those required for IL-2, suggesting a more efficient, and perhaps more physiologically relevant, means by which to enhance CD16-mediated NK cell activity. Indeed, IL-12 has been shown to be highly effective in the enhancement of NK cell lytic activity following CD16 stimulation due to its ability to increase levels of cytolytic enzymes and enhance granule exocytosis $(32,33)$. Work by Ortaldo et al. reveals that NK cells pretreated with IL-2 or IL-12 can undergo apoptosis following crosslinking of CD16 (34).

Our data demonstrate that both IL-2 and IL-12 can elicit NK cell cytokine production in the present model. However, IL-12 appears to be about tenfold more effective than IL-2 in this regard and did not induce NK cell apoptosis at the concentrations used in our model (data not shown). IL-12 is produced during the immune response to both infectious stimuli and malignant cells and is likely available to NK cells encountering Ab-coated targets. The pattern of NK cell cytokine secretion presented herein would likely have profound effects on the anti-tumor immune response. For example, IFN- $\gamma$ can affect tumor cell proliferation and antigen presentation. TNF- $\alpha$ potentiates immune cell recruitment and cellular cytotoxicity, while the chemokines GM-CSF and MIP- $1 \alpha$ can enhance APC function and T cell chemotaxis, respectively. (35-37). The ability of IL-12 to enhance NK cell ADCC and to induce the rapid secretion of a broad array of immunomodulatory cytokines suggests that its coadministration with anti-tumor Ab's represents a useful anti-cancer strategy.

Importantly, even tumor cells with low-level HER2 expression induced cytokine secretion by NK cells when costimulated with adequate amounts of IL-12, suggesting the existence of a potent synergistic signal provided by FcR crosslinking and IL-12R activation. We have shown that both IL-12-mediated STAT4 signaling and FcR-mediated protein tyrosine kinase (PTK) signaling were involved in the induction of cytokine secretion. Recent observations of crosstalk among signaling pathways involving FcR and IL-12R lend support to the data presented herein. Of note, Walker et al. demonstrated that ligation of CD28 in the presence of IL-12 resulted in the synergistic production and enhanced stabilization of IFN- $\gamma$ transcript within NK cells (38). Simultaneous ligation of CD28 and Fc $\gamma$ RIII has also been shown to upregulate transcription of the $I F N-\gamma$ gene within $\mathrm{NK}$ cells, suggesting an interaction between downstream mediators of Fc $\gamma$ RIII and other signaling pathways (39). Moreover, several studies have described a regulatory interplay between the Src and Syk families of nonreceptor PTKs, essential elements in both the FcR- and IL-12R-mediated signaling cascades. Ting et al. have shown that early Fc $\gamma R$-initiated activation of the PTK lck, a member of the Src family, results in the subsequent regulation of Syk family PTKs, essential mediators of FcR-mediated cellular events (40). Furthermore, IL-12R activation has been shown to induce tyrosine phosphorylation of lck in human NK cells (41). Thus, simultaneous activation of the FcR and IL-12R signaling pathways may lead to synergistic interactions between the Src and Syk family PTKs. Indeed, both pathways are known to involve downstream activation of phospholipase $\mathrm{C}-\gamma$, the Ras/MAP kinase cascade, and cell survival mechanisms via the PI 3 -kinase/Akt cascade (40-42). It remains to be determined, however, whether the observed synergy reported herein is due to the enhanced activation of commonly employed signaling components or to unique actions of the FcR and IL-12R signaling pathways on cytokine gene promoter elements.

Depletion of monocytes from the PBMC population severely attenuated IFN- $\gamma$ secretion by NK and T cells stimulated with IL-12 plus Herceptin-coated tumor cells. This effect was not seen in cocultures stimulated with IL-2. The importance of endogenous production of monokines for optimal secretion of IFN- $\gamma$ by NK and T cells has been well reported within the context of the innate immune response $(43,44)$. Infectious stimuli will induce the monocyte/macrophage compartment to secrete IL-12 and other cytokines that bind to constitu- 
tively expressed receptors on NK cells and stimulate the production of IFN- $\gamma$, the prototypic macrophage-activating factor. This intimate connection between NK cells and monocytes allows for amplification and maturation of the immune response. Monocyte secretion of IL-12 in conjunction with $\mathrm{Ab}$ secretion by $\mathrm{B}$ cells has been suggested by Skok et al., who demonstrated that antigenspecific, dendritic cell-derived IL-12 can promote B cell induction of Th2 differentiation and enhancement of humoral immunity (45). Thus, our results may reflect a normal pathway that is important for the removal of Abcoated targets. Interestingly, in our hands, monocytes enhanced NK cell secretion of IFN- $\gamma$ in response to IL-12 alone (see Figure 7a), suggesting a distinct need for monocytes for the adequate production of cytokines by NK cells. Indeed, results of recent studies indicate that activation of NK cells following exposure to LPS or bacterial DNA is mediated by APC-derived IL-12, and that direct contact between APCs and NK cells is important for this response $(46,47)$. Within the context of tumor immunity, Rodriguez-Calvillo et al. have shown that intratumoral IL-12 is largely responsible for the immunotherapeutic effects of NK cell activation in a CT-26 experimental tumor model (48).

It has been hypothesized that the anti-tumor effects of Herceptin therapy are most likely mediated via direct effects on the tumor. Binding of $\mathrm{mAb}$ to the extracellular domain of the HER2 receptor might alter tumor cell growth by one of several different mechanisms, including downregulation of HER 2 expression, altered receptor phosphorylation and signaling, blockade of ligand-induced receptor activation, and/or interaction of HER2 with other members of the EGF-R family (5, 8). However, Clynes et al. recently showed that the antitumor effects of Herceptin in a murine xenograft model of breast cancer required the expression of functional FcR by immune effector cells (10). Our data suggest that, in addition to the direct effects of Herceptin on tumor growth, it may be possible to enhance the response of the innate immune system to Herceptincoated tumor cells via the administration of IL-12.

Herceptin has been previously administered in combination with immunomodulatory cytokines such as GM-CSF and IL-2 $(49,50)$. Although these combinations have shown promise in preclinical models of malignancy, the final results of phase I and phase II clinical trials are still pending. Recently, investigators have developed modifications to the Herceptin molecule itself, resulting in bispecific molecules that recognize HER2 in conjunction with CD3, CD16, CD64, or B7-1, in hopes of mediating better immune stimulation (51-54). These chimeric proteins have been effective in colocalizing the appropriate immune effectors to tumor sites in mice bearing breast cancer xenografts. However, the ultimate success of these agents will depend upon their ability to induce the migration of adequate numbers of activated immune effectors to the tumor site. More recently, $\mathrm{Ab}$ fusion molecules have been developed in which anti-HER2 Ab's are linked to potent cytokines such as RANTES, IL-2, GM-CSF, and even IL-12 (55-57). However, Cavallo et al. have reported that systemic IL-12 administration in a murine model of spontaneously metastatic mammary adenocarcinoma is markedly more effective than locally injected IL-12 (58). Systemic IL-12 enhanced prompt recruitment and activation of leukocytes capable of producing proinflammatory cytokines, CTL activation, and destruction of tumor-associated microvessels by polymorphonuclear cells. Indeed, preliminary results of our ongoing phase I trial, in which patients with HER2-overexpressing tumors are given systemic systemic IL-12 with Herceptin, suggest that combination therapy is effective in the induction of IFN- $\gamma$ secretion by NK and T cells and that cytokine production correlates positively with clinical response (59).

In summary, we have demonstrated that NK cells produce large amounts of immunostimulatory cytokines such as IFN- $\gamma$, TNF- $\alpha$, MIP- $1 \alpha$, and GM-CSF in response to costimulation with Herceptin-coated HER2-overexpressing tumor cells and IL-12. Our in vitro data suggest that this unique cytokine profile is critically dependent upon two distinct signals relayed from the cell surface to the nucleus via FcyRIII and IL-12R, resulting in dramatic synergy for cytokine production. In addition, we have provided evidence suggesting an important interaction between NK cells and monocytes in the observed cytokine response. Coadministration of these two factors to tumor-bearing mice induced significant cytokine production in vivo as well. These data provide a strong rationale for the administration of IL-12 in combination with Herceptin for the treatment of HER2-overexpressing malignancies and yield insight into the immune mechanisms that might be activated when cytokines are given in conjunction with anti-tumor Ab's.

\section{Acknowledgments}

The authors would like to thank M. Anghelina and N. Dakappagari for their experimental advice and thoughtful discussions during the course of this study. This work was supported by NIH grants CA-68326, CA-68458, and P30 CA-16058 and an Ohio State University Department of Surgery seed grant.

1. Ross, J.S., and Fletcher, J.A. 1998. The HER-2/neu oncogene in breast cancer: prognostic factor, predictive factor, and target for therapy. Stem Cells. 16:413-428.

2. Revillion, F., Bonneterre, J., and Peyrat, J.P. 1998. ERB-B2 oncogene in human breast cancer and its clinical significance. Eur. J. Cancer. 34:791-808.

3. Orr, M.S., O’Connor, P.M., and Kohn, K.W. 2000. Effects of c-erbB2 overexpression on the drug sensitivities of normal human epithelial cells. J. Natl. Cancer Inst. 92:987-994.

4. Sarup, J.C., et al. 1991. Characterization of an anti-p185HER2 monoclonal antibody that stimulates receptor function and inhibits tumor cell growth. Growth Regul. 1:72-82.

5. Stancovski, I., et al. 1991. Mechanistic aspects of the opposing effects of monoclonal antibodies to the ERBB2 receptor on tumor growth. Proc. Natl. Acad. Sci. USA. 88:8691-8695.

6. Baselga, J., et al. 1996. Phase II study of weekly intravenous recombinant anti-p $185^{\text {her } 2}$ monoclonal antibody in patients with her $2 /$ neu-overexpressing metastatic breast cancer. J. Clin. Oncol. 14:737-744.

7. Pegram, M.D., et al. 1998. Phase II study of receptor-enhanced chemosensitivity using recombinant humanized anti-p185HER2/neu monoclonal antibody plus cisplatin in patients with HER2/neu-overex- 
pressing metastatic breast cancer refractory to chemotherapy treatment J. Clin. Oncol. 16:2659-2671.

8. Baselga, J., and Albanell, J. 2001. Mechanisms of action of anti-HER2 monoclonal antibodies. Ann. Oncol. 12:S35-S41.

9. Sliwkowski, M.X., et al. 1999. Nonclinical studies addressing the mechanism of action of trastuzumab (Herceptin). Semin. Oncol. 26:60-70.

10. Clynes, R.A., Towers, T.L., Presta, L.G., and Ravetch, J.V. 2000. Inhibitory Fc receptors modulate in vivo cytotoxicity against tumor targets. Nat. Med. 6:443-446.

11. Robertson, M.J., and Ritz, J. 1990. Biology and relevance of human natural killer cells. Blood. 76:2421-2438.

12. Carson, W.E., et al. 2001. Interleukin-2 enhances the natural killer cell response to Herceptin-coated Her2/neu-positive breast cancer cells. Eur. J. Immunol. 31:3016-3025.

13. Lieberman, M.D., Sigal, R.K., Williams, N.N., and Daly, J.M. 1991. Natural killer cell stimulatory factor (NKSF) augments natural killer cell antibody-dependent tumoricidal response against colon carcinoma cell lines. J. Surg. Res. 50:410-415.

14. Gately, M.K., et al. 1998. The interleukin-12/interleukin-12 receptor system: role in normal and pathologic immune responses. Ann. Rev. Immunol. 16:495-521.

15. Tannenbaum, C.S., et al. 1996. Cytokine and chemokine expression in tumors of mice receiving systemic therapy with IL-12. J. Immunol. 156:693-699.

16. Haicheur, N., et al. 2000. Cytokines and soluble cytokine receptor induction after IL-12 administration in cancer patients. Clin. Exp. Immunol. 119:28-37

17. Nanni, P., et al. 2001. Combined allogeneic tumor cell vaccination and systemic IL-12 prevents mammary carcinogenesis in HER-2/neu transgenic mice. J. Exp. Med. 194:1195-1205.

18. Chen, Y., et al. 2001. Induction of ErbB2/neu-specific protective and therapeutic anti-tumor immunity using genetically modified dendritic cells: enhanced efficacy by co-transduction of gene encoding IL-12. Gene Ther. 8:316-323.

19. Yao, L., et al. 1999. Contribution of natural killer cells to inhibition of angiogenesis by interleukin-12. Blood. 93:1612-1621.

20. Carter, P., et al. 1992. Humanization of an anti-p185 HER2 antibody for human cancer therapy. Proc. Natl. Acad. Sci. USA. 89:4285-4289.

21. Jones, R.G., and Landon, J. 2002. Enhanced pepsin digestion: a novel process for purifying antibody $\mathrm{F}\left(\mathrm{ab}^{\prime}\right) 2$ fragments in high yield from serum. J. Immunol. Methods. 263:57-74.

22. Liu, Y., el-Ashry, D., Chen, D., Ding, I.Y., and Kern, F.G. 1995. MCF-7 breast cancer cells overexpressing transfected c-erbB-2 have an in vitro growth advantage in estrogen-depleted conditions and reduced estrogen-dependence and tamoxifen-sensitivity in vivo. Breast Cancer Res. Treat. 34:97-117.

23. Penichet, M.L., et al. 1999. In vivo properties of three human HER2/neuexpressing murine cell lines in immunocompetent mice. Lab. Anim. Sci. 49:179-188.

24. Thierfelder, W.E., et al. 1996. Requirement for Stat4 in interleukin-12mediated responses of natural killer and T cells. Nature. 382:171-174

25. Carson, W.E., et al. 1994. Interleukin-15 is a novel cytokine which activates human natural killer cells via components of the interleukin-2 receptor. J. Exp. Med. 180:1395-1403.

26. Andersen, B.L., et al. 1998. Stress and immune responses after surgical treatment for regional breast cancer. J. Natl. Cancer Inst. 90:30-36.

27. Scott, S.D. 1998. Rituximab: a new therapeutic monoclonal antibody for non-Hodgkin's lymphoma. Cancer Pract. 6:195-197.

28. Oliver, J.M., Burg, D.L., Wilson, B.S., McLaughlin, J.L., and Geahlen, R.L. 1994. Inhibition of mast cell Fc epsilon R1-mediated signaling and effector function by the Syk-selective inhibitor, piceatannol. J. Biol. Chem. 269:29697-29703

29. Cooper, M.A., Fehniger, T.A., and Caligiuri, M.A. 2001. The biology of human natural killer-cell subsets. Trends Immunol. 22:633-640.

30. Cassatella, M.A., et al. 1989. Fc gamma R(CD16) interaction with ligand induces $\mathrm{Ca} 2+$ mobilization and phosphoinositide turnover in human natural killer cells. Role of $\mathrm{Ca} 2+$ in Fc gamma(CD16)-induced transcription and expression of lymphokine genes. J. Exp. Med. 169:549-567.

31. Sahin, U., Kraft-Bauer, S., Ohnesorge, S., Pfreundschuh, M., and Renner, C. 1996. Interleukin-12 increases bispecific-antibody-mediated natural killer cell cytotoxicity against human tumors. Cancer Immunol. Immunother. 42:9-14.

32. Aste-Amezaga, M., D’Andrea, A., Kubin, M., and Trinchieri, G. 1994 Cooperation of natural killer cell stimulatory factor/interleukin-12 with other stimuli in the induction of cytokines and cytotoxic cell-associated molecules in human T and NK cells. Cell. Immunol. 156:480-492.

33. Bonnema, J.D., et al. 1994. Cytokine-enhanced NK cell-mediated cytotoxicity: positive modulatory effects of IL-2 and IL-12 on stimulusdependent granule exocytosis. J. Immunol. 152:2098-2104.

34. Ortaldo, J.R., Mason, A.T., and O'Shea, J.J. 1995. Receptor-induced death in human natural killer cells: involvement of CD16. J. Exp. Med. 181:339-344.

35. Gobin, S.J., and van den Elsen, P.J. 2000. Transcriptional regulation of the MHC class Ib genes HLA-E, HLA-F and HLA-G. Hum. Immunol. 61:1102-1107.

36. Yoon, S.J., et al. 1998. Synergistic anti-tumor effects with co-expression of GM-CSF and IFN-gamma in tumors. Int. J. Cancer. 77:907-912.

37. Prevost-Blondel, A., Roth, E., Rosenthal, F.M., and Pricher, H. 2000. Crucial role of TNF-alpha in CD8 T cell-mediated elimination of 3LL-A9 Lew carcinoma cells in vivo. J. Immunol. 164:3645-3651.

38. Walker, W., Aste-Amezaga, M., Kastelein, R.A., Trinchieri, G., and Hunter, C.A. 1999. IL-18 and CD28 use distinct molecular mechanisms to enhance NK cell production of IL-12-induced IFN-gamma. J. Immunol. 162:5894-5901.

39. Cheung, J.C., Koh, C.Y., Gordon, B.E., Wilder, J.A., and Yuan, D. 1999. The mechanism of activation of NK-cell IFN-gamma production by ligation of CD28. Mol. Immunol. 36:361-372.

40. Ting, A.T., et al. 1995. Interaction between lck and syk family tyrosine kinases in Fc gamma receptor-initiated activation of natural killer cells. J. Biol. Chem 270:16415-16421.

41. Pignata, C., et al. 1995. Phosphorylation of src family lck tyrosine kinase following interleukin-12 activation of human natural killer cells. Cell. Immunol. 165:211-216.

42. Manciulea, M., et al. 1996. Divergent phosphotyrosine signaling via Fc $\gamma$ RIIIA on human NK cells. Cell. Immunol. 167:63-71.

43. Yoshida, A., et al. 2001. IL-18-induced expression of intercellular adhesion molecule-1 in human monocytes: involvement in IL-12 and IFN-gamma production in PBMC. Cell. Immunol. 210:106-115.

44. Tominaga, K., et al. 2000. IL-12 synergizes with IL-18 or IL-1beta for IFN-gamma production from human T cells. Int. Immunol. 12:151-160.

45. Skok, J., Poudrier, J., and Gray, D. 1999. Dendritic cell-derived IL-12 promotes B cell induction of Th2 differentiation: a feedback regulation of Th1 development. J. Immunol. 163:4284-4291.

46. Amakata, Y., et al. 2001. Mechanism of NK cell activation induced by coculture with dendritic cells derived from peripheral blood monocytes. Clin. Exp. Immunol. 124:214-222.

47. Chace, J.H., Hooker, N.A., Mildenstein, K.L., Krieg, A.M., and Cowdery, J.S. 1997. Bacterial DNA-induced NK cell IFN-gamma production is dependent on macrophage secretion of IL-12. Clin. Immunol. Immunopathol. 84:185-193.

48. Rodriguez-Calvillo, M., et al. 2002. Upregulation of natural killer cells functions underlies the efficacy of intratumorally injected dendritic cells engineered to produce interleukin-12. Exp. Hematol. 30:195-204.

49. van Ojik, H.H., Repp, R., Groenewegen, G., Valerius, T., and van de Winkel, J.G. 1997. Clinical evaluation of the bispecific antibody MDXH210 (anti-Fc gamma RI $x$ anti-HER-2/neu) in combination with granulocyte-colony-stimulating factor (filgrastim) for treatment of advanced breast cancer. Cancer Immunol. Immunother. 45:207-209.

50. Fleming, G.F., et al. 1999. A phase I CALGB trial of recombinant human anti-HER2 monoclonal antibody plus low-dose interleukin2 in solid tumors. Proc. Am. Soc. Clin. Oncol. 18:184a. (Abstr. 710)

51. Shalaby, M.R., et al. 1992. Development of humanized bispecific antibodies reactive with cytotoxic lymphocytes and tumor cells overexpressing the HER2 protooncogene. J. Exp. Med. 175:217-225.

52. Weiner, L.M., et al. 1995. Phase I trial of 2B1, a bispecific monoclonal antibody targeting c-erbB-2 and $\mathrm{Fc}$ gamma RIII. Cancer Res 55:4586-4593.

53. Valone, F.H., et al. 1995. Phase Ia/Ib trial of bispecific antibody MDX210 in patients with advanced breast or ovarian cancer that overexpresses the proto-oncogene HER-2/neu. J. Clin. Oncol. 13:2281-2292.

54. Gerstmayer, B., Hoffmann, M., Altenschmidt, U., and Wels, W. 1997. Costimulation of T-cell proliferation by a chimeric B7-antibody fusion protein. Cancer Immunol. Immunother. 45:156-158.

55. Penichet, M.L., Dela Cruz, J.S., Shin, S.U., and Morrison, S.L. 2001. A recombinant IgG3-(IL-2) fusion protein for the treatment of human HER2/neu expressing tumors. Hum. Antibodies. 10:43-49.

56. Dela Cruz, J.S., Trinh, K.R., Morrison, S.L., and Penichet, M.L. 2000 Recombinant anti-human HER2/neu IgG3-(GM-CSF) fusion protein retains antigen specificity and cytokine function and demonstrates antitumor activity. J. Immunol. 165:5112-5121.

57. Peng, L.S., Penichet, M.L., and Morrison, S.L. 1999. A single-chain IL-12 IgG3 antibody fusion protein retains antibody specificity and IL-12 bioactivity and demonstrates antitumor activity. J. Immunol. 163:250-258

58. Cavallo, F., et al. 1999. Immune events associated with the cure of established tumors and spontaneous metastases by local and systemic interleukin-12. Cancer Res. 59:414-421.

59. Parihar, R. et al. 2001. A phase I trial of Herceptin and interleukin-12 in patients with HER2-overexpressing malignancies. Proc. Am. Soc. Clin. Oncol. 20:258a. (Abstr. 1031) 